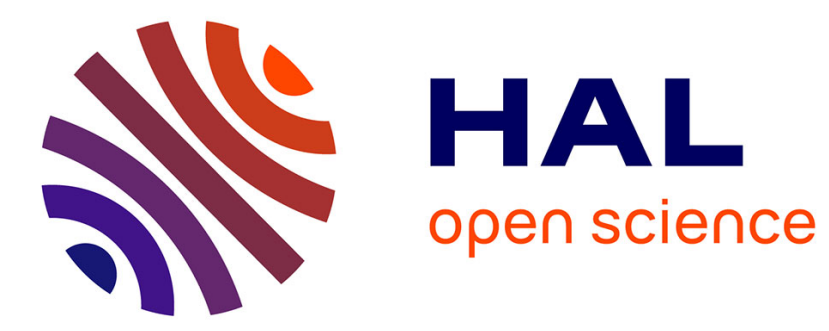

\title{
Problèmes liés à la datation des sols fossiles par le carbone 14.
}

Georgette Delibrias

\section{To cite this version:}

Georgette Delibrias. Problèmes liés à la datation des sols fossiles par le carbone 14.. Bulletin de l'Association française pour l'étude du quaternaire, 1979, 16 (1), pp.35 - 42. 10.3406/quate.1979.1344 . hal-03408832

\section{HAL Id: hal-03408832 \\ https://hal.science/hal-03408832}

Submitted on 29 Oct 2021

HAL is a multi-disciplinary open access archive for the deposit and dissemination of scientific research documents, whether they are published or not. The documents may come from teaching and research institutions in France or abroad, or from public or private research centers.
L'archive ouverte pluridisciplinaire HAL, est destinée au dépôt et à la diffusion de documents scientifiques de niveau recherche, publiés ou non, émanant des établissements d'enseignement et de recherche français ou étrangers, des laboratoires publics ou privés. 


\section{Problèmes liés à la datation des sols fossiles par le carbone 14.}

\section{Georgette Delibrias}

\section{Abstract}

Radiocarbon dating of fossil soils sometimes sets a difficult problem of interpretation because of the complexity of dynamic processes of organic carbon in the soil. Yet, fiability of dates mainly depends on the validity of the samples.

\section{Résumé}

La datation par le carbone 14 des sols fossiles par leur matière organique pose souvent de délicats problèmes d'interprétation qui sont liés à la complexité des phénomènes dynamiques du carbone dans le sol. Cependant la fiabilité des résultats obtenus dépend surtout de la validité des prélèvements eux-mêmes.

\section{Citer ce document / Cite this document :}

Delibrias Georgette. Problèmes liés à la datation des sols fossiles par le carbone 14.. In: Bulletin de l'Association française pour l'étude du quaternaire, vol. 16, n¹-2, 1979. pp. 35-42;

doi : https://doi.org/10.3406/quate.1979.1344

https://www.persee.fr/doc/quate_0004-5500_1979_num_16_1_1344

Fichier pdf généré le 06/11/2020 


\section{PROBLÈMES LIÉS A LA DATATION DES SOLS FOSSILES PAR LE CARBONE 14 \\ par G. DELIBRIAS*}

\section{RESUME}

La datation par le carbone 14 des sols fossiles par leur matière organique pose souvent de délicats problèmes d'interprétation qui sont liés à la complexité des phénomènes dynamiques du carbone dans le sol. Cependant la fiabilité des résultats obtenus dépend surtout de la validité des prélèvements eux-mèmes.

\section{ABSTRACT}

Radiocarbon dating of fossil soils sometimes sets a difficult problem of interpretation because of the complexity of dynamic processes of organic carbon in the soil. Yet, fiability of dates mainly depends on the validity of the samples.

\section{INTRODUCTION}

C'est Libby qui dès 1946 a recconu l'existence du Carbone 14 dans la nature et proposé de l'utiliser comme moyen de datation de vestiges d'origine organique, particulièrement en archéologie. Plus tard, Broecker en 1956 et de Vries en 1958 ont les premiers essayé d'appliquer cette méthode à la détermination de l'âge des sols fossiles en datant leur matière organique. Depuis, de très nombreuses datations ont été effectuées sur des sols.

Le carbone 14 est formé en haute altitude par l'action des neutrons secondaires d'origine cosmique sur l'azote atmosphérique. Rapidement oxydé, il se transforme en gaz carbonique, $\left(\mathrm{CO}_{2}\right)$ qui se mélange au gaz carbonique de l'air ; il est ensuite fixé par les plantes au cours de la photosynthèse au même titre que le carbone stable $\left(98,99 \%\right.$ de ${ }^{12} \mathrm{C}+1,11 \%$ de $\left.{ }^{13} \mathrm{C}\right)$.

Les végétaux vivants présentent ainsi le même rapport ${ }^{14} \mathrm{C} /{ }^{12} \mathrm{C}$, donc la même radioactivité que l'atmosphère. Après la mort, l'équilibre qui existait à la suite des échanges constants avec l'atmosphère est rompu et la radioactivité du carbone 14 du carbone végétal décroît lentement en fonction du temps : la moitié des atomes ${ }^{14} \mathrm{C}$ présents disparaissent après chaque période, soit tous les 5570 ans. En utilisant la loi exponentielle de décroissance, on peut en mesurant la radioactivité résiduelle de débris organiques,
$I$, en déduire le temps, $t$ (en années), qui s'est écoulé depuis la mort.

$$
I=I_{0} e^{-\frac{\ln 2}{5570} t}
$$

$I_{O}$ est l'activité du carbone moderne pris comme référence.

Les possibilités de datation par le ${ }^{14} \mathrm{C}$ s'étendent couramment jusqu'á $\mathbf{4 0 . 0 0 0}$ ans environ.

Ainsi en mesurant la teneur en ${ }^{14} \mathrm{C}$ d'un arbre fossile par exemple, on détermine la date de sa mort, à condition toutefois de prélever les dernières couches annuelles si l'on ne veut pas faire une erreur correspondant à l'âge de l'arbre lui-même.

La datation d'un sol fossile est un problème beaucoup plus délicat : sa matière organique a souvent une origine et une histoire complexe ; le rapport entre le carbonne daté et le $\mathrm{CO}_{2}$ atmosphérique à l'époque oú le sol était en activité, est parfois difficile à établir. De plus les risques de pollution depuis l'enfouissement sont nombreux, surtout dans le cas de sols très anciens. Ces raisons ont conduit les pessimistes à mettre en doute les âges ${ }^{14} \mathrm{C}$ des sols ou même à les rejeter systématiquement. Les optimistes pensent que, malgré ces difficultés, il y a grâce aux paléosols des possibilités d'étude et de datation des évènements climatiques dans le passé que 
l'on se doit de ne pas rejeter ; chaque cas doit cependant être soigneusement examiné afin de définir le degré de confiance qu'il est possible d'accorder à l'âge ${ }^{14} \mathrm{C}$ obtenu.

Après avoir précisé ce que l'on appelle âge d'un sol actuel et âge d'un sol fossile, nous examinerons les problèmes posés par la pollution des sols anciens. Il s'agit ici des sols, au sens pédologique du terme, c'est-à-dire de la partie supérieure du sol qui contient la matière organique.

Le problème de la datation des sols par les carbonates ou les nodules ne sera pas discuté ici ; sauf peut-être dans le cas particulier des régions arides non calcaires, la méthode par le C-14 est alors difficilement utilisable. L'on pourra consulter à ce sujet les travaux de J.M. Bowler et H.A. Polach (1971).

\section{AGE DUN SOL ACTUEL}

Dans un sol en activité les débris organiques s'accumulent et se décomposent simultanément et constituent la couche humifère superficielle. Entre les deux phénomènes, acccumulation et décomposition, s'établit un équilibre qui dépend essentiellement du type de végétation, des conditions climatiques et de la nature du sol ; il conduit à des sols superficiels d'épaisseur variable où la matière organique est plus ou moins abondante.

\section{Evolution de la matière organique $\left(^{*}\right)$}

La décomposition des débris végétaux qui tombent sur le sol, ainsi que les racines qu'il contient, s'effectue selon deux processus différents :

- l'un, rapide, est surtout fonction de l'activité biologique liée aux conditions du milieu ; il conduit à la transformation directe du carbone de la matière végétale en gaz carbonique : c'est la minéralisation.

- l'autre, beaucoup plus lent est constitué par l'ensemble des phénomènes physico-chimiques qui aboutissent à la formation de nouvelles molécules organiques complexes, les composés humiques. Ces composés se minéralisent à leur tour en donnant peu à peu du gaz carbonique. C'est l'humification.

Le schéma est le suivant :

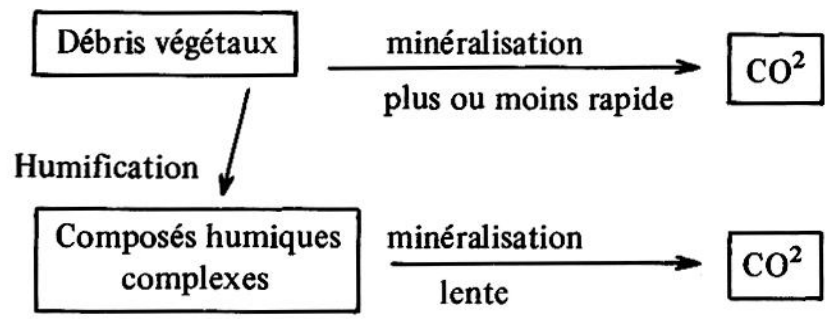

(*) Se reporter à l'ouvrage de Duchaufour (1965).
Ces 2 processus de la dégradation de la matièr organique se produisent en même temps dans le sol, l'un ou l'autre se trouvant plus ou moins favorisé suivant les conditions du milieu. Lorsque l'humification est prépondérante l'on a alors des sols épais et riches en humus où l'on trouve tous les composés humiques intermédiaires, en cours de minéralisation.

Il s'ensuit que la matière organique du sol est constituée par un mélange complexe de plusieurs composés organiques chimiquement différents, de nature d'ailleurs encore mal définie: il est souvent difficile de trouver un procédé chimique permettant de les extraire sélectivement.

Le traitement par les réactifs alcalins permet de séparer plusieurs fractions:

- les acides fulviques, solubles en solution alcaline, et non précipitables par les acides. Ils forment avec les acides aminés, qui sont hydrolysables par $\mathrm{HCl}$, la fraction organique qui est le plus facilement minéralisée.

- les acides humiques solubles en solution alcaline et précipitables par $\mathrm{SO}_{4} \mathrm{H}_{2}$.

- l'humine qui est la matière organique insoluble dans les réactifs alcalins.

Les acides humiques, surtout lorsqu'ils sont liés à l'argile, et l'humine constituent les formes les plus stables de la matière organique.

Ainsi avant d'être restitué à l'atmosphère, le carbone fixé par les végétaux se trouve stocké dans le sol pendant des temps variables suivant:

- la nature du composé humique,

- la profondeur dans le sol : la réalimentation en matière organique fraîche se faisant en surface par les végétaux, la teneur en matière organique décroît en fonction de la profondeur,

- le type de sol considéré : son humidité et sa température, son $\mathrm{pH}$, la nature de la roche mère déterminent ses caractéristiques, en particulier la vitesse de minéralisation de la matière organique.

\section{Age de la matière organique d'un sol actuel}

A cause de la diversité en âge et en quantité de ses différents composants, l'âge de la matière organique des sols ne peut jamais être un âge réel : il ne peut être qu'un âge moyen pondéré, qui résulte de la moyenne de l'activité des composés non minéralisés qui subsistent en quantités variées dans le sol. L'âge ne peut donc représenter qu'un âge moyen minimal pour la matière organique du sol (Geyh et al, 1971, Perrin et al, 1964). Notons que cette valeur prend un sens d'autant plus pré. cis que l'on peut définir quelle fraction organique ou quel niveau a été daté : en effet, l'âge de la matière organique d'un horizon superficiel n'est pas le méme que 
celui d'un horizon profond. Il sera encore différent si l'on date la matière organique totale ou si l'on procède à des séparations chimiques et si l'on date la totalité du sol plutôt qu'un niveau bien défini en épaisseur.

La difficulté d'attribuer un âge à un sol actuel apparaît bien dans l'exemple suivant :

Lors d'une étude effectuée au Canada, Campbell et al (1968) ont obtenu un "âge" de $870 \pm 50$ ans pour le carbone total d'un sol en activité. Les différentes fractions humiques ont donné après séparation, des "âges" de 25 ans pour les produits de l'activité bactérienne et les acides aminés et de 250 à 1500 ans pour les composés organiques plus résistants.

Ce n'est que dans le cadre d'une étude de la dynamique du carbone du sol que l'on procède à la mesure systématique de l'"âge" des différentes fractions de la matière organique : on arrive ainsi à préciser sous quelles formes et pour quelles durées se trouve stocké le carbone qui constitue la biomasse. Dans la pratique, pour dater un sol on ne détermine que l'âge des fractions organiques les plus stables : l'humine et les acides humiques.

Dans l'exemple précédent, l'âge du sol canadien étudié est de 1500 ans : c'est l'âge des composés organiques les plus résistants, c'est-à-dire le temps de résidence le plus long du carbone organique dans ce sol ; les 2 termes âges et temps de résidence sont employés pour désigner la même valeur. Cet âge de 1500 ans ne donne aucune indication sur l'époque de mise en place du sol qui est probablement très antérieure, mais c'est la valeur qui en est la plus proche.

L'âge de la mise en place du sol est d'autant plus proche de l"âge" du sol que le temps de résidence du carbone organique $y$ est grand.

Aux latitudes moyennes, en Europe par exemple, l'on sait que les sols actuels se sont peu à peu installés depuis la dernière glaciation, mais le temps de résidence moyen du carbone y est, le plus souvent, court : il excède rarement 1200 à 1400 ans.
C'est ainsi que dans la région parisienne, on a daté à 400 ans la fraction la plus ancienne, d'un sol forestier, à l'orée d'un bois de chênes et de châtaigniers (G.D., S. Nakhla, 1967) (fig. 1).

Par contre, des âges de 5.000 à 6.000 ans sont trouvés couramment pour la matière organique des horizons profonds des chernozems et des sols de steppe d'Allemagne et d'Europe Centrale. Dans certains cas, on a mesuré des temps de résidence moyens supérieurs à 10.000 ans pour les horizons les plus profonds $(2 \mathrm{~m}$ à $2,80 \mathrm{~m}$ ) ; ils pourraient alors probablement représenter la mise en place du sol (Scharpenseel, 1977). D'après Gerasimov, 1971 , ces âges très anciens s'expliqueraient par la fixation en profondeur de la matière organique sur l'argile ; elle se trouverait ainsi à l'abri de toute activité microbienne et de tout lessivage.

Dans le cas des andosols, on a également conservation de la matière organique à la suite de formation d'un complexe très stable avec les alumino-silicates. Ce type de sol se développe essentiellement sur cendres et ponces volcaniques : il a été reconnu en France, dans le Massif Central, par Moinereau, 1965. D'après J.M. Hetier 1975, le temps moyen de résidence y serait de l'ordre de 4.000 ans à 6.000 ans.

Dans les podzols, la matière organique lessivée ainsi que le fer à partir des niveaux superficiels du sol, s'accumulent dans l'horizon profond $\mathrm{B}$. Son âge moyen est de 2.000 à 3.000 ans; c'est le cas des podzols étudiés en France dans le Sud-Ouest et le Bassin Parisien (G. Delibrias, Dutil, 1966, G. Delibrias, S. Nakhla, 1972) et dans les Vosges (B. Hassko et al, 1974), en Angleterre (Perrin, Willis et Hodge, 1964) ainsi qu'en Allemagne du Sud (Scharpenseel et Pietig, 1971). La constance des dates trouvées pour ces horizons quelles que soient les régions considérées est remarquable.

Il ne semblerait pas cependant, d'après les études détaillées de nombreux profils, faites en Allemagne (Scharpenseel, 1972) que cet âge corresponde à une période de podzolisation, mais au temps moyen de résidence du carbone dans l'horizon $B$.

\begin{tabular}{|c|c|c|c|c|c|c|c|c|}
\hline \multirow[b]{2}{*}{ Année } & \multicolumn{3}{|c|}{$0-3 \mathrm{~cm}$} & \multicolumn{3}{|c|}{$3-9 \mathrm{~cm}$} & \multicolumn{2}{|c|}{$9-20 \mathrm{~cm}$} \\
\hline & $\begin{array}{l}\text { Feuilles } \\
\delta{ }^{14} \mathrm{C} \%\end{array}$ & $\begin{array}{l}\text { Humine } \\
{ }_{\delta}{ }^{14} \mathrm{C} \%\end{array}$ & $\begin{array}{c}\text { Acides } \\
\text { humiques } \\
\delta{ }^{14} \mathrm{C} \%\end{array}$ & $\begin{array}{l}\text { Racines } \\
\qquad \delta{ }^{14} \mathrm{C} \%\end{array}$ & $\begin{array}{l}\text { Humine } \\
\qquad{ }^{14} \mathrm{C} \%\end{array}$ & $\begin{array}{c}\text { Acides } \\
\text { humiques } \\
{ }_{\delta}{ }^{4} \mathrm{C} \%\end{array}$ & $\begin{array}{l}\text { Humine } \\
\delta{ }^{14} \mathrm{C} \%\end{array}$ & $\begin{array}{c}\text { Acides } \\
\text { humiques } \\
\text { Age (années) }\end{array}$ \\
\hline Août 1964 & $+37,4$ & +28 & +15 & $+50,6$ & +9 & 0 & 0 & $380 \pm 100$ \\
\hline Aoũt 1965 & +62 & +29 & $+17,5$ & +58 & $+12,5$ & 0 & 0 & $420 \pm 100$ \\
\hline Août 1966 & $+73,5$ & +40 & $+20,5$ & +62 & +28 & 0 & 0 & $390 \pm 100$ \\
\hline
\end{tabular}

Fig. 1. - Livolution de l'activité spécifique du carbone dans les divers constituants de la matière organique d'un sol forestier de la Région Parisienne (Gif-sur-Yvette) en fonction du temps : de 0 à $9 \mathrm{~cm}$ l'activité des débris végétaux et des 2 fractions étudiées est égale $\left(\delta{ }^{14} \mathrm{C} \%=0\right)$ ou supérieure $\left(\delta{ }^{14} \mathrm{C} \% \geqslant 0\right)$ à celle du carbone moderne référence; dans ce dernier cas elle reflète l'augmentation d'activité due au ${ }^{14} \mathrm{C}$ artificiel des bombes. Ces résultats indiquent un "turn-over" rapide de la matière organique dans ce niveau ; de 9 à $20 \mathrm{~cm}$, l'âge de $\mathbf{4 0 0}$ ans qui peut être attribué aux acides humiques montre que c'est cette fraction organique qui a ici le temps de résidence le plus long. 


\section{AGE D'UN SOL FOSSILE}

Un sol fossile est un sol qui a cessé tout échange de carbone avec l'atmosphěre, par suite soit d'une variation climatique, soit d'un évènement quelconque, qui a fait cesser toute végétation. Les sols fossiles sont le plus souvent conservés grâce à un recouvrement par des matériaux rapportés.

N'étant plus réapprovisionné en carbone actuel riche en ${ }^{14} \mathrm{C}$ par les végétaux vivants, l'ensemble du carbone du sol fossilisé, de la même façon que le carbone d'un tronc d'arbre qui s'y trouverait enterré, va voir sa radioactivité décroître peu à peu en fonction du temps.

La mesure du rapport ${ }^{14} \mathrm{C} /{ }^{12} \mathrm{C}$ de la matière organique du sol indique le temps qui s'est écoulé depuis la fossilisation plus l'âge (ou temps de résidence) moyen de la matière organique du sol au moment de son activité.

L'âge de la matière organique est d'autant plus proche de l'âge vrai que le temps de résidence moyen est court par rapport au temps écoulé depuis la fossilisation.

Ainsi dans le cas de paléosols vieux de 20.000 ans un temps de résidence moyen du carbone de 400 ans est négligeable. Mais s'il s'agit de la fossilisation de sols pour lesquels le temps de résidence de la matière organique est de 5.000 ans par exemple, l'on peut arriver à des âges apparents de fossilisation vieillis de façon importante. $\mathrm{Si}$ l'on veut dater l'évènement qui a mis fin à l'activité du sol : retombés volcaniques ou dépôt de loess par exemple, on doit déduire une estimation du temps de résidence moyen de la matière organique du sol prélevé.

L'on peut arriver à un âge de fossilisation plus proche de la réalité en datant la partie supérieure du paléosol, si cela est possible. Cependant les limites en épaisseur des sols fossiles sont souvent mal définies et leur teneur en carbone organique très basse ; on est souvent obligé de faire des prélèvements dans toute l'épaisseur du sol et l'on obtient de ce fait une valeur moyenne qui introduit une imprécision difficile à chiffrer.

L'on voit apparaître ici l'importance des observations faites sur le terrain et des conditions de prélèvement. Ce point capital sera évoqué de nouveau à propos des problèmes de pollution. Il sera en particulier souhaitable d'avoir des informations d'ordre pédologique et de savoir si l'on a affaire à un sol à temps de résidence important. La teneur en matière organique de l'horizon daté peut donner une indication intéressante ; des teneurs en carbone organique importantes $>1 \%$, par exemple) peuvent faire soupçonner l'existence d'un sol à temps de résidence long.

Considérons un profil étudié par Brousse et al. (1969), dans la Chaîne des Puys, au lieu-dit "La Cheire de Mercoeur". Il est constitué (fig. 2) par un horizon organique fossile de $15 \mathrm{~cm}$ intercalé entre 2 couches de retombées volcaniques. Le tout, reposant sur une coulée de lave, est

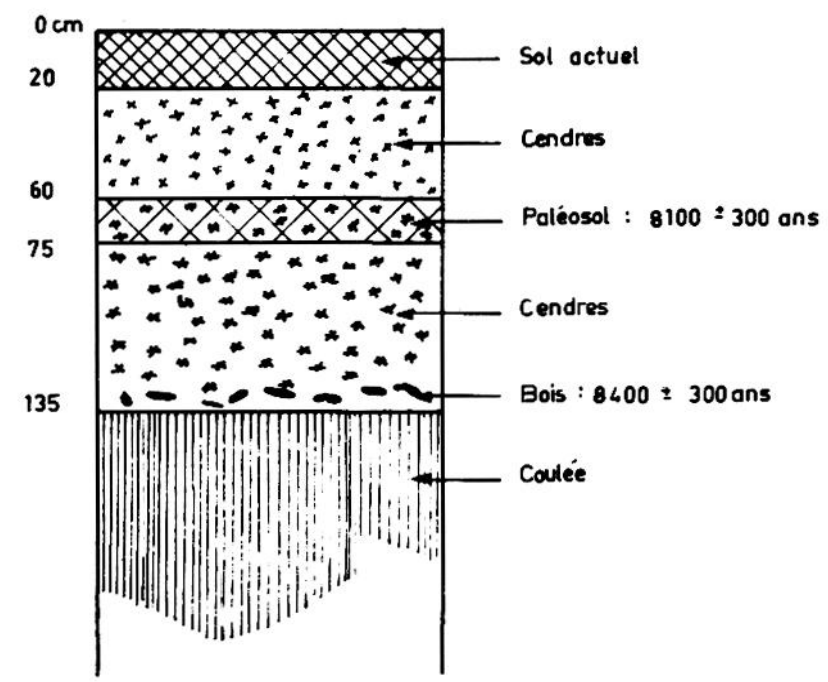

Fig. 2. - Coupe de la "Cheire de Mercoeur" dans la Chaîne des Puys (Massif Central)

coiffé par un sol actuel de $20 \mathrm{~cm}$ d'épaisseur. Des fragments de bois, emballés dans les cendres de la couche inférieure ont été datés de $8.400 \pm 170$ ans (Gif-1498) alors que la matière organique du sol sus-jacent a donné un âge de $8.100 \pm 300$ ans (Gif-1497). La faible différence entre les âges mesurés, même en tenant compte des erreurs statistiques qui affectent chacune des mesures, laisse peu de temps pour le développement d'un sol aux dépens de la première couche de cendres et pour la mise en place de la végétation. Ainsi ces résultats, tout en étant chronologiquement bien ordonnés posaient un problème d'interprétation un peu difficile.

Sur un profil très proche de celui-ci, Hétier (1975) a identifié et étudié un andosol actuel ; le temps de résidence moyen de sa matière organique totale a été mesuré et trouvé de 3.000 ans dans l'horizon profond (40$50 \mathrm{~cm}$ ) et de 1.600 ans dans l'horizon supérieur $A_{1}$ $(20$ à $30 \mathrm{~cm})$. Si l'on envisage la possibilité que le sol fossile de 8.100 ans soit également un andosol fossilisé, l'âge du niveau supérieur de cendres se trouverait ainsi considérablement rajeuni. La teneur en carbone organique, bien que faible $(0,7 \%)$ ne permet pas d'éliminer cette éventualité car il s'agit d'un horizon fossilisé.

Les datations ${ }^{14} \mathrm{C}$ sur la matière organique de sols fossilises à temps de résidence long ne peuvent alors donner que des indications chronologiques et non des valeurs précises : l'incertitude sur le temps de résidence dans le niveau étudié ne permet qu'une estimation de la correction à effectuer.

\section{TECHNIQUE DE PREPARATION DES SOLS}

Au laboratoire, chaque prélèvement de sol est d'abord examiné soigneusement à l'aide d'une loupe binoculaire ; les racines et radicelles qui peuvent s'y trouver sont soi- 
gneusement triées à la pince. Néanmoins leur élimination complète est difficile; dans certains cas elle est à priori impossible ; l'échantillon est alors impropre à la datation, surtout s'il s'agit d'un sol très ancien.

Les traitements chimiques qui suivent cette préparation manuelle varient selon que l'on veut étudier la dynamique d'un sol en activité ou dater un horizon fossile.

Dans le premier cas, l'on sépare les différentes fractions de l'humus du sol : acides aminés, acides fulviques, acides humiques et humine ; précisons qu'il faut disposer de quantités suffisantes pour permettre la mesure de l'âge de chacun d'eux.

Nous n'exposerons pas en détail ces différentes operations. Dans le cas d'un horizon fossile la préparation chimique est standard dans tous les laboratoires ; elle comprend :

- un traitement dans l'acide chlorhydrique $\mathrm{N} / 10$ pour éliminer toute trace de calcaire : il est plus ou moins prolongé et répété suivant la nature du sol.

- un traitement dans une solution ammoniacale à $1 \%$ dans laquelle les acides humiques passent en solution en donnant une coloration brune. Cette operation, souvent laborieuse, est répétée jusqu'à extraction complěte ; plus de 10 traitements sont quelques fois nécessaires.

- la fraction insoluble dans la solution ammoniacale constitue l'humine. Elle est centrifugée, rincée jusqu'á $\mathrm{pH} 7$, et récupérée en même temps que la partie minérale décarbonatée du sol. On brûle alors le sol total décarbonaté ; le carbone de l'humine est oxydé et récupéré sous forme de gaz carbonique. C'est cette dernière fraction, généralement la plus abondante, qui est datée.

Lorsque les acides humiques sont présents en quantité suffisante, ils sont précipités par l'acide sulfurique, et récupérés pour être datés également.

Une des difficultés techniques de la préparation des sols fossiles est due à leur pauvreté en carbone organique qui conduit souvent à traiter 1 ou $2 \mathrm{~kg}$ de sol.

\section{POLLUTION DES SOLS FOSSILES}

Les sols fossiles constituent un milieu éminement polluable ; ce sont des sols dont la matière organique a progressivement disparu et dont la teneur en carbone organique résiduel est souvent très inférieure à $1 \%$. De plus, ils sont souvent très anciens, de ce fait leur.carbone ne présente plus qu'une radioactivité très faible. Pour ces deux raisons, ils sont particulièrement sensibles à toute contamination : le moindre apport de carbone organique exogène récent donc riche en ${ }^{14} \mathrm{C}$ peut conduire à une augmentation relative importante de la teneur en carbone et à une augmentation appréciable du rapport ${ }^{14} \mathrm{C} /{ }^{12} \mathrm{C}$ du carbone du sol étudié.
La principale cause de rajeunissement des sols est la présence de racines. Malgré les précautions prises lors de la préparation des échantillons, il est difficile d'être sûr de les avoir éliminées totalement.

Par ailleurs, des eaux de percolation peuvent entraîner en profondeur, de la matière organique soluble récente. Ce phénomène a également pu se produire à un moment quelconque de l'histoire du sol.

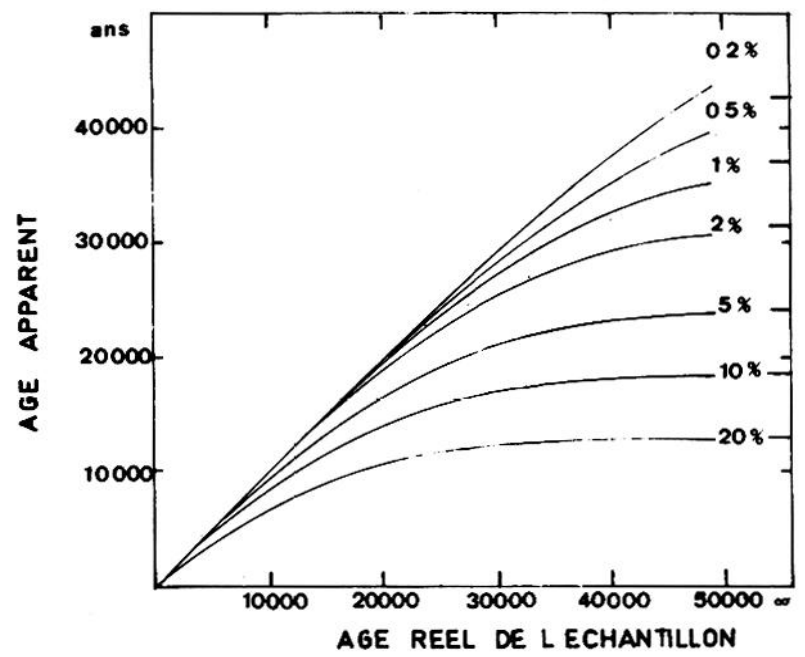

Fig. 3. - Age apparent en fonction de l'âge réel d'un échantillon par suite de contamination par des quantités variées de carbone moderne.

La figure 3 montre le rajeunissement subi théoriquement par un échantillon en fonction d'une pollution variable par du carbone moderne.

L'on voit ainsi qu'un sol de $\mathbf{3 0 . 0 0 0}$ ans peut être rajeuni de 8.000 ans par un apport de $4 \%$ de carbone moderne et qu'un autre, dont l'âge est au-delà des possibilités de datation par le ${ }^{14} \mathrm{C}$ peut avoir un âge ap. parent de 31.000 ans s'il est pollue par $2 \%$ de carbone moderne.

Les risques de vieillissement par apport de carbone ancien sont par contre peu probables; ce genre de pollution est éliminé par les traitements chimiques qui détruisent toute trace de calcaire. De plus, il affecte peu les "vieux" échantillons : un apport de $5 \%$ en poids conduit à un vieillissement de 400 ans ; un tel vieillissement est peu sensible pour des âges de $\mathbf{3 0 . 0 0 0}$ ans par exemple. Des études récentes du rapport ${ }^{13} \mathrm{C} /{ }^{12} \mathrm{C}$ du carbone organique dans des sols calcaires ont montré par ailleurs, que les craintes de voir les plantes assimiler en partie sous forme de bicarbonates le carbone provenant des roches sédimentaires anciennes n'étaient pas fondées et qu'aucun vieillissement de la matière organique n'est à envisager en région calcaire (Scharpenseel, 1977).

Indépendamment des traitements que subit l'échantillon en laboratoire pour éliminer les éventuelles pollutions, un certain nombre de précautions doivent être prises en particulier au moment du prélèvement ; 
elles tendent à accroître la validité des prélèvements eux-mêmes et à permettre de mieux juger du degré de confiance qu'il est possible d'attribuer à l'âge finalement obtenu :

- toutes les observations qui peuvent être faites sur le terrain à propos des possibilités de pollution sont précieuses : relevé précis de la stratigraphie, profondeur de pénétration des racines provenant du sol superficiel, présence de circulation latérale d'eau qui nous paraît également pouvoir être à l'origine du rajeunissement de certains niveaux ;

- les coupes doivent être rafraîchies avant tout prélèvement : un rajeunissement par suite de l'existence de végétation ou d'une activité biologique qui s'étendrait latéralement, dans le cas de coupes longuement exposées à l'air est à craindre.

- on renoncera, quel que soit l'intérêt du problème, à des prélèvements dans des niveaux trop superficiels ou présentant des traces de racines.

Par ailleurs, la datation respective des acides humiques et de la fraction insoluble peut aider à la compréhension de l'histoire de la matière organique du sol depuis sa fossilisation, elle peut, en particulier, mettre en évidence un éventuel rajeunissement de la fraction soluble par apport d'acides humiques récents ; l'âge le plus ancien est alors considéré comme le plus proche de l'âge réel. Une grande différence d'âge entre les deux fractions est l'indice d'une pollution récente.

Un autre test de pollution récente est également possible. D'après Scharpenseel (1977) lors du traitement acide du sol en vue d'éliminer le calcaire, des composés organiques tels que les acides aminés sont extraits ; il s'agit là d'une fraction organique très jeune, c'est-à-dire à temps de résidence court. La présence de ces composés serait la preuve que le sol fossile étudié est réalimenté en carbone actuel et qu'il n'est donc pas conservé à l'abri des pollutions.

Il est peu probable qu'un sol fossile enterré soit pollué sans que les niveaux sus-jacents le soient également, les pollutions récentes provenant le plus souvent du sol superficiel en activité. L'étude par le ${ }^{14} \mathrm{C} d u$ profil complet et non d'un horizon isolé paraît être un moyen de mettre en évidence une pollution éventuelle ; toutefois le transport de matière organique récente peut être trop faible pour être décelé dans les niveaux supérieurs. Si la fraction organique soluble dans la solution ammoniacale est présente dans le niveau de recouvrement directement en contact avec le paléosol, c'est le signe d'un transport encore actuel de matière organique du sol superficiel qui risque d'avoir atteint le paléosol étudié.

Nous donnerons ici l'exemple de la datation d'un sol fossile en forêt de Fontainebleau, présentant de bonnes conditions de conservation et de prélèvement, et suffisamment riche en matière organique pour permettre l'un des tests proposé ci-dessus.
Des carrières ouvertes dans la plaine de Chanfroy, en forêt de Fontainebleau, font apparaître des coupes verticales dans les formations superficielles reposant sur le sable stampien en place. Cette plaine est le résultat du comblement d'une dépression par accumulation des débris provenant de l'évolution des versants, à la suite de phénomènes de gélifraction et de solifluction (Puyfaucher et Collin, 1971).

L'un des profils, particulièrement étudié, présentait un horizon organique de 5 à $10 \mathrm{~cm}$, à une profondeur de 2,40 m environ : il s'agit plus probablement d'un dépôt de marécage dans une cuvette que d'un paléosol. Ce dépôt argilo-sableux contenait de la matière organique en quantité relativement abondante : $0,6 \%$ de carbone, sous forme d'humine et $0,3 \%$ sous forme d'acides humiques. Reposant sur un niveau de sable contenant à sa base des galets de grès calcaire, il est surmonté d'une "grève" sableuse constituée de débris anguleux dans une matière sableuse. Le tout est coiffé par le sol actuel (fig. 4).

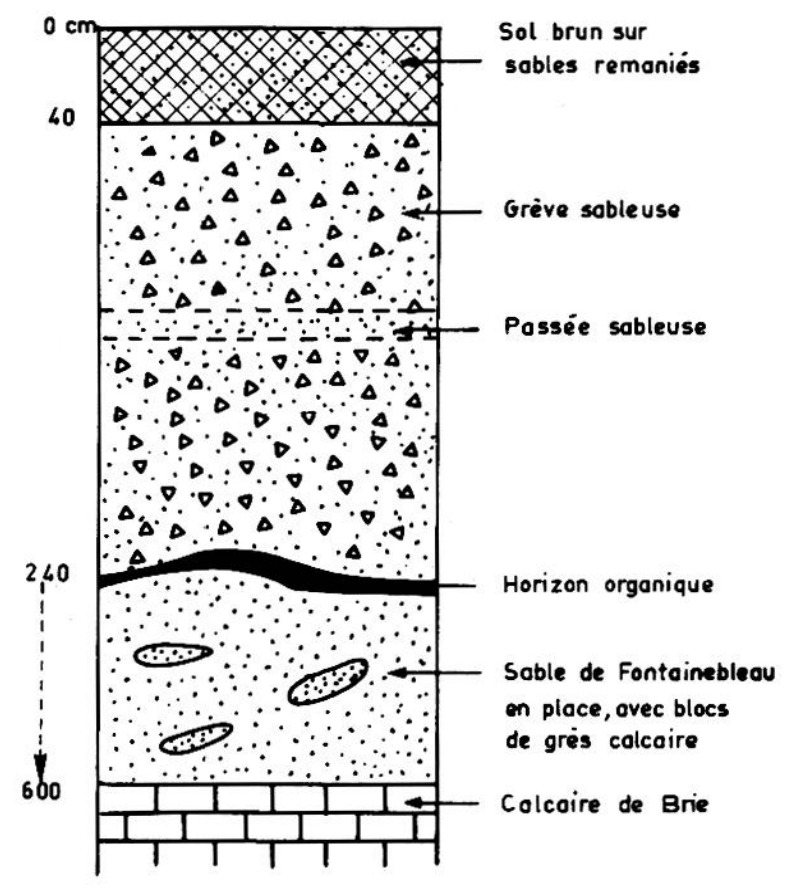

Fig. 4. - Coupe de la plaine de Chanfroy, Forêt de Fontainebleau.

La matière organique de l'horizon fossile a été datée en utilisant séparément la fraction insoluble et la fraction soluble, puisque toutes deux étaient présentes en quantité suffisante ; les âges obtenus sont les suivants :

$23.350 \pm 500$ ans pour l'humine (Gif-2510)

$22.860 \pm 500$ ans pour les acides humiques (Gif-2913)

Un autre prélèvement, dans le même niveau, mais distant de $50 \mathrm{~m}$, avait conduit déjà un âge de $22.100 \pm$ 700 ans (Gif-1905) sur la fraction soluble seulement. La convergence de ces résultats, obtenus pour deux prélèvements différents, ainsi que l'absence contrôlée de carbone sous forme soluble ou insoluble dans les 
niveaux sus-jacents nous incitent à considérer cette datation de la matière organique comme significative de l'âge de l'horizon organique lui-même.

La position profonde de ce niveau à l'abri de la pénétration des racines, la fraîcheur des coupes effectuees au fur et à mesure de l'exploitation de la carrière a permis des prélèvements à l'abri, semble-t-il des contaminations récentes.

\section{CONCLUSION}

La datation des sols fossiles présente un très grand intérêt pour l'étude de la géologie et de la paléoclimatologie du quatemaire récent.

L'utilisation $d u{ }^{14} \mathrm{C}$ pour la détermination des âges pose le problème du comportement très complexe des différents composés organiques et a déjà conduit à de nombreuses études pédologiques. Il n'est cependant pas possible de prétendre que tous ces phénomènes sont actuellement très bien connus et il est vrai que l'on peut se trouver parfois en présence de résultats encore inexplicables.

La datation d'un sol n'est pas une datation "absolue" comme peut l'être la datation de charbon ou d'os provenant d'un site archéologique bien caractérisé ; c'est une date qui, à cause du temps de résidence de la matière organique dans le sol ne peut théoriquement être considérée que comme minimale.

Par ailleurs, la contamination de sols fossiles même profondément enterrés est toujours possible et quelque fois difficile à mettre en évidence.

Chaque cas fera donc l'objet de critiques sévères concernant essentiellement les conditions de gisement.

On recherchera par ailleurs à replacer le sol daté dans un contexte chronostratigraphique ou paléoclimatologique de façon à renforcer la validité des âges obtenus.

En dépit des multiples difficultés d'interprétation qui ont été évoquées, la datation de la matière organique des sols par le ${ }^{14} \mathrm{C}$ apporte cependant une information jusqu'à présent irremplaçáble, à condition toutefois de s'entourer de toutes les précautions qui s'imposent et qui sont inhérentes à la nature même du matériau daté.

Manuscrit reçu en septembre 1977

\section{BIBLIOGRAPHIE}

[1] Libby W.F., 1946. - Phys. Rev., 69, 671.

[2] Broeker W.S., Kulp J.L. and Tucek C.S., 1956. Lamont Natural Radiocarbon measurements III, Science, 124, p. 154.
[3] De Vires H.L., 1958. - Radiocarbon dates for upper Eem and Wurm-interstadial samples Eiszeitalter Gegenwart, 9 - 10-17 (Cit. Scharpenseel, 1977)

[4] Bowler J.M. and Polach H.A., 1971. - Radiocarbon analyses of soil carbonates : an evaluation from paleosols in southeastern Australia. in : Paleopedology - origin, nature and dating of paleosols, 99-108, Jerusalem 1971.

[5] Duchaufour P., 1965. - Précis de Pédologie, Masson et $\mathrm{Cie}, \mathrm{Ed}$. Paris

[6] Campbell C.A., Paul E.A., Rennie D.A. and Mc Callum K.J., 1969. - Applicability of the carbon dating method of analysis to soil humus studies". Soil Sci, 104 : 217-224.

[7] Geyh. M.A., Benzler J.H. and Roeschmann C., 1971. - Problems of dating pleistocene and holocene soils by radiometric methods. In Paleopedology : Origin, nature and dating of paleosols, 63-75 - Jérusalem, 1971.

[8] Perrin R.M.S., Willis E.H., and Hodge C.A.M., 1964. - Dating of humus podzols by residual radiocarbon. Nature, 202-4928, 165-166.

[9] Nakhla S.M., Delibrias G., 1967. - Utilisation du C-14 d'origine thermonucléaire pour l'étude de la dynamique du carbone dans le sol. Radioactive dating and methods of low level counting, Proc. Symp. IAEA, 169 Monaco, 1967.

[10] Scharpenseel H.W. and Schiffmann, 1977. Soil Radiocarbon Analysis and Soil Dating Geophysical Review, sous presse.

[11] Gerastimow I.P., 1971. - Nature and originality of paleosols In Paleopedology : Origin, Nature and Dating of Paleosol, 15-27, Jérusalem.

[12] Moinereau J., 1965. - Observations pédologiques sur les sols du Massif volcanique des Coirons. Bull. AFES, 6-7, 188-223.

[13] Hetier J.M., 1975. - Formation et évolution des andosols en climat temperé. Thèse, Nancy 1975.

[14] Delibrias G., Dutil P., Juste C., 1966. - Premiers résultats de mesure d'âge de la matière organique de l'horizon d'accumulation des podzols humiques développés sur le sable des Landes. C.R. Acad. Sc. Paris, tome 263, p. 1300-1302.

[15] Delibrias G., Nakhla M.S., 1972. - Study of some podzol levels from France, by ${ }^{14} \mathrm{C}$ dating of humic matter : Proceeding of 8th International Conference on Radiocarbon dating, New Zealand.

[16] Hassko B., Guillet B., Jaegy R. and Coppens R., 1974. Nancy Natural Radiocarbon Measurements. III. Radiocarbon, vol 16, pp. 118-130. 
[17] Perrin R.M.S., Willis E.H., Hodge C.A.M., 1964 .Dating of humus podzols by residuel radiocarbon activity. C.A.M., 1964, Nature, vol. 202, p. 165 . 166.

[18] Scharpenseel H.W. and Pietig F., 1971. - University of Bonn Natural Radiocarbon Measurements. IV. Radiocarbon, vol. 13, pp. 189-212.

[19] Scharpenseel H.N., 1972. - Natural radiocarbon measurements on humic substances in the light of carbon cycle estimates : Proc. Int. Meet. Humic substances, Nienwersluis, Pudoc, Wageningen.

[20] Brousse R., Delibrias G., Labeyrie J. et Rudel A., 1969. - Eléments de chronologie des éruptions de la chaîne des Puys. Bull. de la Soc. Geol. de France, XI, p. 770-93.

[21] Puyfaucher A. et Collin F., 1971. - Les grèves des plaines de Chanfroy, de la Feuillardière et du Cul du Chaudron (Forêt de Fontainebleau). Bull. Assoc. Fr. pour l'Etude du Quaternaire, vol 4, p. 241-249. 